\title{
Vertical Germanium Nanowire Photodetectors with Suspended Graphene Top Contact
}

\author{
Shi-Qiang $\mathrm{Li}^{1^{*}}$, Jacopo Frigerio ${ }^{2}$, Daniel Chrastina ${ }^{2}$, Giovanni Isella ${ }^{2}$, Amit Solanki $^{3}$, Wuzhou Song ${ }^{4}$, \\ Changxi Zheng ${ }^{5}$, and Kenneth B. Crozier, ${ }^{1,4 *}$ \\ ${ }^{I}$ Department of Electrical and Electronic Engineering, University of Melbourne, Victoria 3010, Australia \\ ${ }^{2}$ Dipartimento di Fisica del Politecnico di Milano, L-NESS, Polo Regionale di Como, Via Anzani 42, I-22100 Como, Italy \\ ${ }^{3}$ School of Engineering and Applied Sciences, Harvard University, 33 Oxford Street, Cambridge, Massachusetts 02138, USA \\ ${ }^{4}$ School of Physics, University of Melbourne, Victoria 3010, Australia \\ ${ }^{5}$ School of Physics, Monash University, Clayton, Victoria 3800, Australia \\ Author e-mail addresses: shiqiang.li@unimelb.edu.au, kenneth.crozier@unimelb.edu.au
}

\begin{abstract}
We demonstrate photodetectors for visible-to-infrared imaging, comprising vertical germanium nanowires on a silicon substrate, with a suspended graphene layer as the top electrical contact. Measured responsivity spectra show peaks that shift with increasing nanowire diameter.

OCIS codes: (040.5160) Photodetectors; (220.4241) Nanostructure fabrication; (230.5160) Photodetectors;
\end{abstract}

\section{Introduction}

Multispectral imaging captures images in several spectral bands, and thus provides richer information than conventional color imaging. Currently, it is generally performed by combining a photodetector array (image sensor) with filters, e.g. a filter wheel or a filter array. Recent progress in nano-optics however presents an alternative approach, in which the spectral responses of the photodetectors are tailored by nanostructuring. This enables greater integration, removing the need for the filter wheel or filter array. We recently showed that vertically-oriented nanowires (NWs) made of silicon (Si) [1] and germanium (Ge) [2] can exhibit peaks in their absorption spectra, whose positions shift with nanowire diameter. We demonstrated multispectral imaging using this effect, with the Si NWs acting as spectral filters. [3] We furthermore showed that these Si NWs could be made into photodetectors, and made use of their tailorable absorption spectra to demonstrate color imaging. [4] Here we propose and demonstrate a new architecture that presents the opportunity for multispectral image sensors that are formed on Si substrates and operate over the visible to short-wave-infrared (SWIR) range. Our device (Fig. 1(a.i-ii)) consists of vertically-oriented Ge NWs (doped p+ and intrinsic) on a silicon substrate $($ doped $\mathrm{n}+$ ). In our previous Si NW photodetectors, we filled the spaces between the nanowires with an insulator (polymethylmethacrylate, PMMA), and then sputtered on indium tin oxide (ITO) to form the top contact [4]. In Fig. 1(a.iii) we plot simulated spectra of the fractional absorption of light by arrays of Ge NWs with various diameters. We consider NWs embedded in PMMA (Fig. 1(a.iii) left) and in air (Fig. 1(a.iii) right). In both cases, the spectral peaks in absorption shift across the visible-to-SWIR with increasing nanowire diameter. This confirms that this architecture should be suitable for spectrally-resolved imaging in this wavelength range. It can be seen however that the PMMA-embedded NWs have reduced absorption and wider spectral peaks that their air-embedded counterparts (Fig. 1(a.iii)). In this work, therefore, we demonstrate air-embedded NW arrays. These are achieved using suspended graphene as the top electrical contact (Fig. 1(a,i-ii)).

\section{Results and Discussions}

Fabrication begins with a Si wafer, onto which crystalline Ge is formed by low energy plasma-enhanced chemical vapor deposition. NWs are then formed by lithography and etching. A single layer of graphene (goldcoated) is mechanically transferred to the chip, and annealed to ensure electrical contact with the tops of the NWs. The graphene sits on a platform (silicon dioxide or germanium with silicon nitride film) that surrounds the NW array and is formed by deposition, lithography and etching. We fabricate photodetectors (PDs) with and without passivation. The unpassivated devices are termed NW PDs (Fig. 1(b.i-ii)). The passivated devices are termed $p$-NW PDs (Fig. 1(b.iii-iv)), where the prefix " $p$-“ denotes silicon dioxide passivation. The currentvoltage characteristics measured on these devices are shown as Fig. 1(c). While the $p$-NW PD shows rectifying diode behavior, the NW PD with the same geometric parameters does not. It is also found that NW PDs show inconsistent and noisier photo-responsivities, in comparison with the $p$-NW PDs. This suggests the importance of passivation, which to be expected due to the high surface area to volume ratios of the NWs.

The photo-responsivities of $p$-NW PDs ( $\mathrm{SiO}_{2}$ thickness: $80 \mathrm{~nm}$ ) measured at zero bias (Fig. 1(d)) show spectral peaks that red-shift with increasing diameter. This trend is consistent with simulations (Fig. 1(a.iii)). Spectral responsivities are also measured for the NW PDs, with some devices exhibiting spectral peaks that shift with diameter. The NW PDs however behaved inconsistently and we therefore do not present the results here. It can be noted that the responsivity peaks of Fig. 1(d) are quite broad, with full-widths-at-half-maximum (FWHMs) of $\sim 500$ to $700 \mathrm{~nm}$. In Fig. 1(e), we plot the responsivity spectra measured on $p$-NW PDs with different passivating layer thicknesses $(20 \mathrm{~nm}$ and $80 \mathrm{~nm})$. The thinner passivation layer device exhibits only 
marginal improvement in FWHM, and both are much wider than predicted by simulation (Fig.1(f)). A detailed investigation is underway.

We next measure the photoresponse of the $p$-NW PD under reverse bias, with the wavelength of the incident illumination maintained at $\lambda=1026 \mathrm{~nm}$. It can be seen that the photocurrent increases markedly with increasing reverse bias (Fig. 1(g)). This improvement saturates at $\sim-15 \mathrm{~V}$, at which the photocurrent is $\sim 50$ times larger than at zero bias. This improvement is referred to as the "enhancement factor" in Fig. 1(g)). For further increases beyond $-15 \mathrm{~V}$, the photocurrent is seen to fluctuate. We attribute the photocurrent enhancement to the enlargement of the depletion region with reverse bias. To understand this effect, we model the axial electric field $\left(E_{z}\right)$ in the nanowire for different values of reverse bias (Fig. 1(h)). As the reverse bias increases, the region with high electric field increases in size. This is expected to lead to photogenerated electron-hole pairs being more quickly split and extracted, reducing the likelihood of recombination by surface traps. It is possible that the saturation in enhancement factor (at $\sim 15 \mathrm{~V}$ ) corresponds to the electrons and holes reaching saturation velocity. We expect that electron-hole pairs created outside the high field region are strongly affected by surface recombination, with the radial electric field (created by surface traps) drawing the carriers to the surface where recombination then takes place.

\section{Conclusion}

We proposed and demonstrated a new type of multispectral photodetector based on silicon-germanium nanowire devices with graphene as the top contact. Passivation was found to improve performance consistency. We found that operating the device under reverse bias can significantly boost photoresponse.
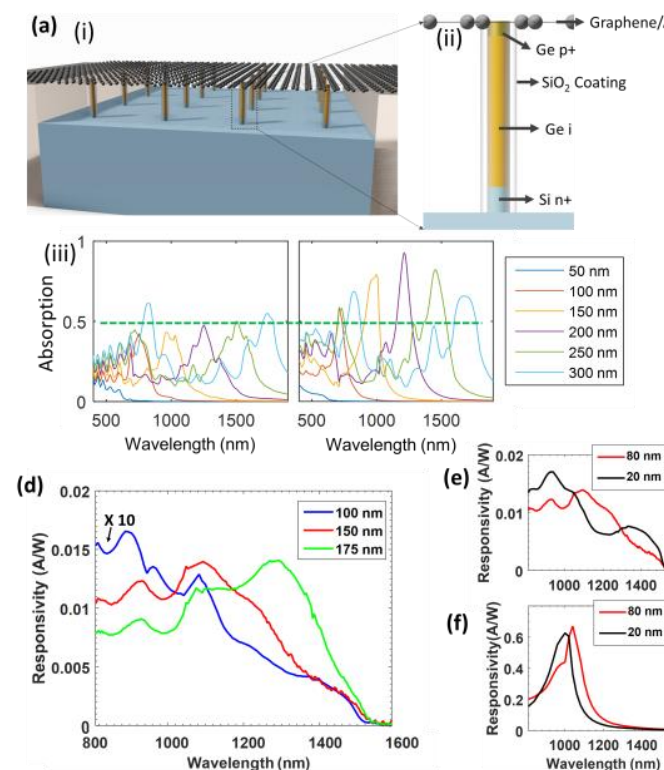

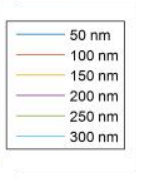

(e)
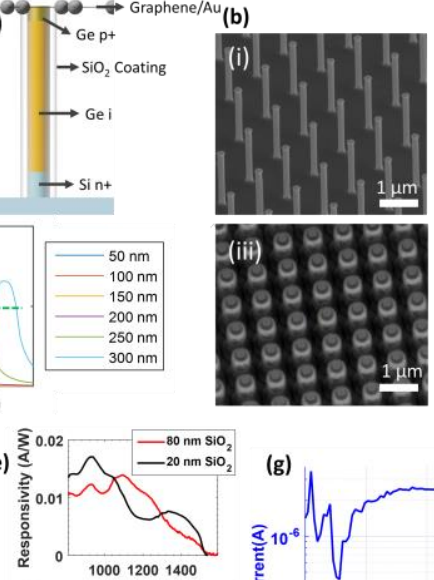

(f)

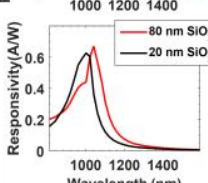

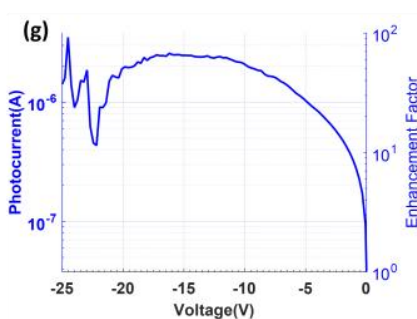

(c)
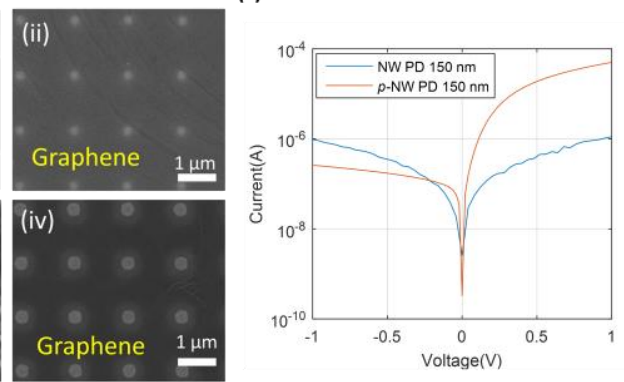

(h)

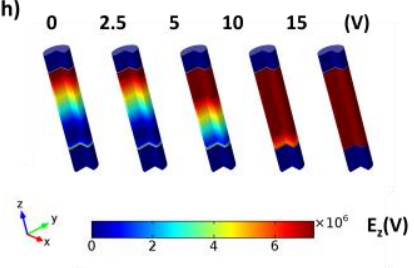

Figure 1. (a) (i). Perspective (ii) side schematic views of Si-Ge nanowire photodetector with graphene as top electrode. Ge p+ layer, Ge intrinsic layer, and etched Si n+ section are 200, $1200 \mathrm{~nm}$, and $300 \mathrm{~nm}$ thick, respectively. (b) (i) and (iii) are tilted scanning electron microscope (SEM) images of unpassivated (NW PD) and passivated ( $p$-NW PD) nanowires before graphene transfer. (ii) and (iv) are topview SEM images of devices after graphene transfer. Nanowires are on a square lattice (period $1500 \mathrm{~nm}$ ). (c). I-V characteristics of NW devices, both with diameter $150 \mathrm{~nm}$. p-NW PD has 80nm thick $\mathrm{SiO}_{2}$. (d). Measured responsivities of $p$-NW PDs of different diameters. (e). Responsivities of $p$-NW PDs with different $\mathrm{SiO}_{2}$ coating thickness, both with diameter $150 \mathrm{~nm}$. (f). Simulated responsivity spectra for arrays of nanowires (150 nm diameter), with different $\mathrm{SiO}_{2}$ coating thickness (assumes unity internal QE). (g). Photocurrent (left-hand axis) and enhancement factor (right-hand axis) vs reverse bias voltage. (f) Internal electric field maps in nanowire at different reverse bias voltages.

\section{Acknowledgements}

Fabrication was performed in part at the Melbourne Centre for Nanofabrication (MCN) of the Australian National Fabrication Facility (ANFF). Funding: Australian Research Council (ARC), VESKI, and the National Science Foundation. (NSF).

\section{References}

[1] K. Seo et al, "Multicolored vertical silicon nanowires," Nano Letters 11, 1851 (2011)

[2] A. Solanki and K.B. Crozier, "Vertical germanium nanowires as spectrally-selective absorbers across the visible-to-infrared," Applied Physics Letters 105, 191115 (2014)

[3] H. Park, and K.B. Crozier, "Multispectral imaging with vertical silicon nanowires," Scientific Reports 3, 2460 (2013)

[4] H. Park et al, "Filter-free image sensor pixels comprising silicon nanowires with selective color absorption,” Nano Lett. 14, 1804 (2014) 


\section{University Library}

\section{- M M N E R VA A gateway to Melbourne's research publications}

Minerva Access is the Institutional Repository of The University of Melbourne

Author/s:

LI, S;Frigerio, J;Chrastina, D;Isella, G;Solanki, A;Song, W;Zheng, C;Crozier, KB

Title:

Vertical Germanium Nanowire Photodetectors with Suspended Graphene Top Contact

Date:

2016

Citation:

LI, S., Frigerio, J., Chrastina, D., Isella, G., Solanki, A., Song, W., Zheng, C. \& Crozier, K. B. (2016). Vertical Germanium Nanowire Photodetectors with Suspended Graphene Top Contact. 2016 CONFERENCE ON LASERS AND ELECTRO-OPTICS (CLEO), OSA. https:// doi.org/10.1364/CLEO_SI.2016.SM4E.7.

Persistent Link:

http://hdl.handle.net/11343/112429 